Correspondence to: Professor Rosalind L Smyth, Division of Child Health, School of Reproductive and Developmental Medicine, University of Liverpool Institute of Child Health, Alder Hey Children's Hospital, Liverpool L12 2AP, UK; r.l.smyth@liv. ac.uk

\begin{abstract}
ystic fibrosis (CF) is the most common life limiting genetic disorder of white populations. There are over 6000 patients in the UK with this condition and at least 30000 in the USA. ${ }^{1}$ This review focuses on the challenges of diagnosis, clinical complications, and management of children with CF.
\end{abstract}

\section{DIAGNOSIS OF CF}

CF is a multisystem disorder with a wide spectrum of clinical manifestations, and patients may present to a range of medical specialists. Table 1 lists some of the clinical features, which may lead to a presentation of CF. The diagnosis can be confirmed by sweat and/or genetic testing. In 2001, the UK's Department of Health announced that newborn screening for CF would be introduced nationally and it is likely that this will be implemented by 2006. By identifying infants with CF at an early age, newborn screening will have a considerable impact on the investigation of children with suspected CF. However, in most areas of the country, for many years there will be a large cohort of children and adults with CF who have not been diagnosed as part of a neonatal screening programme and who will need to be investigated by sweat and/or genetic testing. Sweat testing will also continue to play a role in the investigation of the infant where the screening tests suggest CF.

Sweat chloride $>60 \mathrm{mmol} / \mathrm{l}$ is considered diagnostic of CF. Values of sweat chloride of 40 $60 \mathrm{mmol} / \mathrm{l}$ are considered highly suggestive of CF, and in this situation the sweat test should be repeated and/or genetic testing conducted. Although in CF patients there is a fall in sweat chloride with age, the magnitude of this fall is not sufficient to cause diagnostic confusion, if these criteria are used. ${ }^{2}$ Sweat sodium measurements are less reliable as concentrations of $60-80 \mathrm{mmol} / \mathrm{l}$ are seen in patients without CF, especially in adults. Other indices, or measurements, available from the sweat test have also been evaluated for their diagnostic potential. Some sweat testing systems report only sweat sodium concentrations, which are not, on their own, considered sufficient to differentiate between control and CF populations. Patients with CF usually have a sweat sodium:chloride ratio $>1$, but the value of this ratio as a discriminating measure has not been conclusively demonstrated. Some sweat testing systems measure sweat conductivity alone. Again, there are concerns about whether this can discriminate between CF patients and normal individuals. In general a diagnosis of CF should not be based on sweat conductivity measures alone.

In summary, the diagnosis of CF is made by sweat testing when there is elevation of sweat chloride by $>60 \mathrm{mmol} / \mathrm{l}$ in a patient with one or more clinical features consistent with the CF phenotype, a positive neonatal screening test, or history of CF in a sibling.

\section{Genetic testing for CF}

In a child suspected to have CF, DNA testing for the more common CF transmembrane regulator (CFTR) mutations is a valuable diagnostic test. If two CFTR mutations are identified, this confirms the diagnosis of CF. In all children in whom CF has been diagnosed by sweat testing, identification of the CFTR mutations provides more information concerning potential genotype/ phenotype correlations and can be used for cascade testing of the extended family. DNA may be obtained from mouthwash samples, buccal scrapes, or white blood cells. Following DNA extraction, samples are usually tested using one of the commercially available kits. These should test sufficient gene mutations to account for a high proportion of known gene mutations in the region, or particular ethnic group of the patient. If only one, or none, of the gene mutations are identified, then further testing can be conducted for other common CF genes.

When a diagnosis of CF is confirmed in a child, it is good practice to investigate all other siblings, even those who have no clinical features of the condition.

\section{Newborn screening for CF}

Neonatal screening became feasible with the development, in 1979, of a radioimmunoassay for immunoreactive trypsin (IRT) suitable for use on dried blood spots from newborns. ${ }^{3}$ IRT 
Table 1 Presentation of cystic fibrosis (CF)

- Respiratory

- Chronic or recurrent cough or chest infections

Purulent sputum or haemoptysis

"Asthma" with chest infections

Nasal polyps, chronic sinusitis

- Gastrointestinal

Meconium ileus, meconium plug syndromes

Failure to thrive

Bulky offensive stools

Rectal prolapse

Hypoproteinaemia, oedema

Prolonged neonatal jaundice

$>$ Other

Short stature

Pseudo-Bartter's syndrome

Male infertility

Salty taste when kissed

Sibling with CF

Heat exhaustion

Unexplained clubbing

concentrations are raised in the first few weeks of life in babies with CF. However, in the first week of life, when most newborn blood samples are routinely taken, specificity of a single raised IRT is low. Thus two or three stage protocols based on a second IRT test with or without subsequent DNA analyses have been developed to reduce the false positive diagnoses associated with one stage IRT testing. ${ }^{3}$

Despite acceptable test performance, there is still controversy in some countries about whether neonatal screening for CF should be introduced nationally. ${ }^{4}$ In 1983 the American Cystic Fibrosis Foundation identified a need for further information on the benefits and risks of early diagnosis and treatment before newborn screening could be recommended. ${ }^{5}$ Subsequently, a number of studies have been published from different countries. Dankert-Roelse observed that patients identified by neonatal screening had less pulmonary inflammation and less deterioration of lung function than patients detected on clinical symptoms. ${ }^{6}$ Farrell found that neonatal screening provides the opportunity to prevent malnutrition in infants with CF. ${ }^{7}$ A systematic review $^{8}$ identified two randomised controlled trials of neonatal screening in CF. A meta-analysis was not possible and the reviewers concluded that there was little evidence of either benefit or harm from neonatal screening.

The proposed screening programme in the UK has decided to employ a protocol based on DNA analysis of an initial raised IRT, with a second IRT on samples when only one CFTR mutation is recognised. ${ }^{9}$

\section{CLINICAL FEATURES OF CF}

$\mathrm{CF}$ is a multisystem disorder and clinical progression in an individual patient is unpredictable. With appropriate treatment, the majority of the clinical features, which are evident on presentation, can be corrected or controlled, and most children with CF achieve normal growth with few signs of chest infection and malabsorption.

\section{Respiratory}

The most troublesome symptoms for CF are those related to the chest and around $97 \%$ of deaths in CF are due to pulmonary causes. A persistent cough, often rattly and exacerbated by viral infections, is often the first symptom. Some infants present with a prolonged bronchiolitic type illness and many have recurrent wheezing, suggestive of asthma. In a minority, the wheezing is persistent in the first 1-2 years, gradually improving throughout early childhood. At least $20 \%$ of CF children have coexistent atopic asthma. Sputum expectoration is usually only obvious in older children, although some infants and young children vomit sputum that has been swallowed.

Clinical signs in the chest are often absent in children with CF. In infants with wheezing and hyperinflation there may be Harrison's sulci, an increase in the anterior-posterior diameter of the chest wall, and downward displacement of the liver. In older children, persistent hyperinflation leads to a barrel shaped chest and kyphosis. Finger clubbing develops in parallel with the progression of suppurative lung disease. Chest auscultation may be normal even in the presence of extensive respiratory disease but may reveal an increased expiratory phase, inspiratory and expiratory crackles of varying coarseness, and polyphonic wheezing. Central cyanosis is a very late sign of respiratory disease.

Simple allergy to Aspergillus fumigatus is common in CF but up to $10 \%$ of patients develop allergic bronchopulmonary aspergillosis (ABPA). This is characterised by increased respiratory symptoms with wheezing, fleeting pulmonary shadows, blood and sputum eosinophilia, raised total IgE concentrations, raised IgE and IgG to A fumigatus, and positive A fumigatus sputum cultures. Treatment of this condition is considered in the following section. Mycetomas and invasive aspergillosis can occur in CF but are rare.

\section{Gastrointestinal}

Meconium ileus causes intestinal obstruction in up to $15 \%$ of newborn children with CF. The condition may be detected antenatally by the presence of a "bright bowel" caused by inspissated meconium. After birth, failure to pass meconium, abdominal distension, and bile stained vomiting are characteristic. Abdominal radiographs reveal distended loops of small bowel and ground glass opacification in the lower abdomen. The majority of cases require surgical intervention.

Distal intestinal obstruction syndrome (DIOS), sometimes referred to as "meconium ileus equivalent", has a similar pathophysiology to meconium ileus and occurs in older children and adults. Typically, a faecal mass can be palpated in the right iliac fossa. Occasionally a DIOS mass may form the apex of an intussusception.

Malabsorption caused by pancreatic insufficiency occurs in around $85 \%$ of children with CF. At presentation, affected infants often have a voracious appetite but are slow to gain weight. Failure to thrive is accompanied by steatorrhoea, manifest by the frequent passage of large, greasy, and offensive stools. Distension of the abdomen is a common feature in infants and children who present with CF. A voracious appetite may compensate sufficiently for malabsorption to enable reasonable weight gain in early childhood and diagnosis may be delayed in these children. Recurrent rectal prolapse occurs in up to $10 \%$ of undiagnosed children with CF and may be the sole presenting feature. Inadequate absorption of fat soluble vitamins has occasionally caused symptoms, including bleeding disorders resulting from vitamin $\mathrm{K}$ deficiency, benign intracranial hypertension caused by vitamin A deficiency, and haemolytic anaemia or neurological symptoms caused by vitamin E deficiency. 
Salt deficiency is common at diagnosis and can result in severe hypochloraemic metabolic alkalosis. Undiagnosed CF infants with loose stools and poor weight gain are sometimes treated by substitution of cow's milk with a soya milk based formula. Such children are at risk of developing hypoproteinaemic oedema, anaemia, and essential fatty acid deficiencies.

\section{Liver disease}

A spectrum of liver abnormalities may occur in CF. A few patients with CF have prolonged neonatal jaundice. Hepatomegaly secondary to fatty infiltration is well recognised in malnourished patients but can occur in those who are thriving. Gallstones occur more frequently in people with CF than in the normal population. They are generally asymptomatic and are often an incidental finding on routine ultrasound. Progressive liver involvement, with liver cirrhosis and portal hypertension, occurs in a minority. A recent epidemiological study reported that the clinical presentation of CF related liver disease is relatively uncommon in young children; there is a peak in adolescence (with as many as $20 \%$ of adolescents developing chronic liver disease) and a fall in prevalence over the age of 20 years. ${ }^{10}$

Children with advanced liver disease may develop associated complications including acute variceal haemorrhage, and haematemesis. Haematemesis may be the first presentation of liver disease and may be confused with haemoptysis. It may also cause aspiration and respiratory deterioration.

\section{Diabetes mellitus}

Endocrine pancreatic insufficiency occurs with increasing frequency after the age of 10 years. Blood glucose, urinalysis, and glycosylated haemoglobin measurements do not reliably identify diabetes compared with glucose tolerance tests. ${ }^{11}$ Ketoacidosis is rare. Most CF diabetics benefit from insulin treatment but should not change to a diabetic diet.

\section{Miscellaneous}

Transient non-specific arthritis occurs in some CF patients and most commonly affects the knee and ankle joints. The condition is possibly related to high concentrations of circulating immune complexes. It should be distinguished from hypertrophic pulmonary osteoarthropathy and other causes of arthritis. Oral fluoroquinolones cause joint pains in some patients. There is an increased incidence of gastrointestinal malignancies among CF patients.

\section{TREATMENT OF CF}

In this section evidence concerning treatments has primarily been obtained from The Cochrane Library as either systematic reviews of randomised controlled trials or randomised controlled trials. It should be stressed at the outset that the clinical management of CF involves a multidisciplinary team including medical, nursing, physiotherapy, dietetic, psychology, and social work staff.

\section{Respiratory}

Management of the chest in CF is aimed at the prevention of bacterial infection and colonisation together with prompt treatment of acute exacerbations using antibiotic. Airway clearance using physiotherapy is one of the main features of regular treatment of patients with CF.

One strategy for prevention of infection with Staphylococcus aureus is to use continuous oral anti-staphylococcal treatment from the time of diagnosis. A Cochrane systematic review, which included four randomised controlled trials of a total of 401 children with CF, aged from 0-7 years, found a reduced prevalence of $S$ aureus in the respiratory secretions in children receiving this regimen. ${ }^{12}$ There was no significant difference in the number of isolates of Pseudomonas aeruginosa between groups, though there was a trend towards a lower cumulative isolation rate of $P$ aeruginosa in the prophylaxis group at 2 and 3 years and a trend towards a higher rate from 4-6 years.

Chronic colonisation with $P$ aeruginosa occurs in up to $80 \%$ of adults with CF. ${ }^{13}$ Concern about the association between colonisation with $P$ aeruginosa and worsening respiratory function has led to strategies to eradicate $P$ aeruginosa when it is first identified. In a small randomised controlled trial Valerius et $a l^{14}$ showed that chronic colonisation can be prevented by treatment with a combination of oral and nebulised anti-pseudomonal antibiotics. As there are only limited oral anti-pseudomonal antibiotics available, and development of resistant organisms is an important problem, oral therapy is not an appropriate option for long term antipseudomonal prophylaxis. An alternative, which has been developed over the last 20 years, is the use of antipseudomonal antibiotics delivered in a nebulised form. A systematic review of nebulised anti-pseudomonal antibiotics for CF included 11 trials and a total of 873 participants. ${ }^{15}$ The review suggested that this treatment was associated with an improvement in lung function. There was most information about treatment with tobramycin, which is the most commonly used nebulised anti-pseudomonal antibiotic in North America, but in Europe colistin is most frequently used.

Cross infection between CF patients of pathogenic organisms is now widely reported. It was first demonstrated between CF patients colonised with Burkholderia cepacia. ${ }^{16}$ This was reported to be associated with more rapid deterioration in lung function and some patients experienced an acute severe deterioration and death. Because of this evidence, it is generally accepted within CF clinics and wards that $B$ cepacia post-patients should be segregated from $B$ cepacia negative patients. However, recently it has been shown that a more virulent epidemic form of $B$ cepacia can be spread within a $B$ cepacia cohort of CF patients and that this has also been associated with deaths. This has led to the recognition that policies to "cohort" patients may not be adequate and complete segregation of CF patients harbouring harmful transmissible organisms is the safest practice. ${ }^{17}$

In 1996 Cheng et $_{\text {al }}{ }^{18}$ reported a series of children from one CF centre in whom colonisation by the same $\beta$ lactam resistant stain of $P$ aeruginosa occurred in over $70 \%$ of those colonised with $P$ aeruginosa. This evidence has led to strategies of segregating patients colonised with $P$ aeruginosa from those not colonised, both as inpatients and in outpatient clinics. $B$ cepacia and $P$ aeruginosa are generally only harmful to other CF patients. However, organisms such as methicillin resistant Staphylococcus aureus (MRSA) may colonise the airways of CF patients and if transmitted represent a risk, not only to $\mathrm{CF}$ patients, but to other groups of patients. Individuals with CF who are colonised with such organisms should be strictly isolated when admitted to hospital or seen in the outpatient clinic.

Respiratory exacerbations are treated with oral or intravenous antibiotics, usually for 10-14 days. The choice of these antibiotics is usually based on recent organisms grown 
from the sputum and their sensitivities to antibiotics. There is some controversy about intravenous treatment regimens. For patients colonised with $P$ aeruginosa, a broad spectrum $\beta$ lactam antibiotic, such as an anti-pseudomonal cephalosporin, in combination with an aminoglycoside has been used in a number of centres. It has been suggested that intravenous aminoglycoside treatment, given as a single daily dose, may be as effective with fewer toxic effects than the conventional three times daily dosing, and one large clinical trial, recently completed, suggests that this is the case. $^{19}$

Corticosteroids in high doses are the main treatment for APBA because they are thought to treat the inflammatory and allergic aspects of the condition. An alternative strategy to treating APBA is to reduce or clear the lung of A fumigatus by antifungal treatment. This has been shown to be of some benefit in patients with asthma, but a recent systematic review in CF patients failed to identify any randomised controlled trials in this group. ${ }^{20}$

\section{Physiotherapy}

A number of different physiotherapy techniques are used. ${ }^{21}$ These include postural drainage, percussion and vibration techniques, huffing and directed coughing. The active cycle of breathing technique includes relaxation and breathing control, forced expectoration technique, thoracic expansion exercises, and may also include postural drainage or chest clapping. Devices are used in some physiotherapy regimens and include positive expiratory mask therapy or high pressure positive expiratory masks and oscillating devices such as the flutter/cornet or the oscillator vest. A metaanalysis of chest physiotherapy in CF using sputum weight and pulmonary function as primary outcomes suggested that standard physiotherapy produces greater sputum expectoration than no physiotherapy and that, in addition, exercise was associated with improved lung function results. ${ }^{22}$ There was no difference demonstrated between the modes of physiotherapy used in the treatment of CF.

\section{Anti-inflammatory treatment}

Because persistent inflammation is implicated in the pathophysiology of CF, for many years now strategies for reducing airway inflammation in CF have been investigated. The most powerful anti-inflammatory agents are corticosteroids and a number of trials of long term oral corticosteroid treatment have been conducted in CF; these have been assessed in a systematic review. ${ }^{23}$ This review showed that oral steroids at a dose equivalent to $1 \mathrm{mg} / \mathrm{kg}$ of prednisolone on alternate days were associated with a slowing in the progression of lung disease in CF. This benefit, mainly determined by a change in spirometric lung function, needs to be balanced against the adverse effects observed. Linear growth retardation was seen as early as six months from the start of treatment in the higher dose $(2 \mathrm{mg} / \mathrm{kg})$ group and from two years of treatment in the $1 \mathrm{mg} / \mathrm{kg}$ alternate day treatment group. Other adverse effects including abnormalities in blood glucose and cataracts resulted in early termination of one of the randomised controlled trials. A long term follow up study at 10 years showed that catch-up growth started two years after treatment with oral steroids was stopped. However, alternate day treatment with oral steroids may have resulted in growth impairment up to adulthood in boys but not in girls.
Because of these long term adverse effects of oral steroids, other strategies for delivering anti-inflammatory treatment have been explored. A systematic review, of inhaled corticosteroids in $\mathrm{CF}^{24}$ which included 10 trials, found that current evidence from randomised controlled trials was insufficient to establish whether this treatment was beneficial or harmful. The review recommended further trials to determine whether this treatment was effective and there is currently a trial of withdrawal of inhaled steroid treatment in progress in the UK. A systematic review of non-steroidal antiinflammatory treatment ${ }^{25}$ provided preliminary evidence that these drugs may prevent pulmonary deterioration in patients with mild lung disease caused by CF.

There is some evidence that macrolide antibiotics, while not having direct anti-pseudomonal actions, may be beneficial in CF because of indirect effects, including antiinflammatory effects. A systematic review, which included four trials, found clear evidence of a small but significant improvement in respiratory function following treatment with azithromycin. The largest study employed a three times a week dose and, in that study, treatment with azithromycin was associated with a significant increase in mild (mainly gastrointestinal) adverse events. ${ }^{26}$

\section{Mucolytics}

A number of different treatments have been used to reduce the viscosity of CF sputum and therefore aid its clearance. Most recently the two treatments that have been studied in clinical trials are recombinant human deoxyribonuclease (DNase) and hypertonic saline. In 1990 a recombinant human DNase was produced; this has been commercially available since 1992. This treatment was studied in a very large (over 900 participants) clinical trial in people with CF. ${ }^{27}$ The results produced were exciting because it was the first treatment shown clearly to produce a $>5 \%$ improvement in lung function in CF. However, follow up was for only six months, did not include children under 5 years of age, and excluded patients with severe lung disease. A systematic review of 12 randomised controlled trials representing a total of 2294 participants showed that lung function improved in the treated groups, with significant differences at one month, three months, six months, and two years. The only significant adverse effect was hoarseness. ${ }^{28}$

Nebulised hypertonic saline has been proposed to improve sputum clearance. A systematic review of nine randomised controlled trials ${ }^{29}$ showed that nebulised hypertonic saline improves mucociliary clearance immediately after administration. However, the maximum time for which dates were recorded in these trials was only three weeks. This was therefore insufficient for this treatment to be recommended in CF patients. A trial comparing nebulised DNase, given daily, with nebulised hypertonic saline and DNase given on alternate days in CF has recently reported. ${ }^{29}$ There was no difference between daily and alternate day DNase, but daily DNase showed a significantly greater increase in forced expiratory volume in one second $\left(\mathrm{FEV}_{1}\right)$ over 12 months than hypertonic saline.

\section{Nutritional support}

The main aims of nutritional support in CF are to achieve optimal nutritional status, with normal growth and development throughout childhood. Regular assessment of nutrition, including height and weight measurements by a specialist CF dietician, is recommended. The main strategy is to maximise 
energy intake from food and to ensure adequate pancreatic enzyme supplementation. Additional support is needed for the minority of children in whom this approach is not successful. There are no precise rules about when nutritional interventions are considered, but the dietician will consider whether the child is gaining weight and height satisfactorily along the centiles, the pattern of recent weight gain, the body mass index and its centile, and the percentage ideal weight for height. A systematic review has highlighted the lack of evidence for the efficacy of oral calorie supplements in $\mathrm{CF} .{ }^{31} \mathrm{~A}$ trial, in CF children, is in progress to evaluate this further. Enteral feeding via nasogastric, orogastric, or gastrostomy tubes are considered for children whose nutritional state is unsatisfactory despite dietary advice and/or nutritional supplements. The feeds are commercially available and the range of products includes elemental, semi-elemental, and whole protein feeds. Evidence for the efficacy of these feeds from randomised controlled trials is lacking. ${ }^{32}$

Fat malabsorption in CF means that fat soluble vitamins A, $\mathrm{D}, \mathrm{E}$, and $\mathrm{K}$ will be inadequately absorbed. Routine supplementation of vitamins $\mathrm{A}, \mathrm{D}$, and $\mathrm{E}$ is recommended for all pancreatic insufficient patients with CF. For children over the age of 1 year, the dose recommended for vitamin $\mathrm{A}$ is 4000-10 000 IU daily, vitamin D 400 IU daily, and vitamin E 50-100 mg daily. Plasma concentrations should be measured as part of the annual review and the dose of vitamin adjusted appropriately. Pancreatic sufficient patients should also be monitored by measuring their vitamin values annually, but generally supplements are not required and should only be used if low plasma concentrations are detected.

Patients with CF are at an increased risk of developing vitamin $\mathrm{K}$ deficiency caused by fat malabsorption, bowel salt deficiency, liver disease, and antibiotic treatment. Prolonged prothrombin times are indicative of severe vitamin $\mathrm{K}$ deficiency and supplementation should be used if this is found. Plasma vitamin $\mathrm{K}$ concentrations are an unreliable assessment of status. Vitamin $\mathrm{K}$ is present in many of the multivitamin preparations but additional supplementation can be given in the presence of prolonged prothrombin time, particularly if there is concomitant liver disease.

\section{Pancreatic enzyme replacement therapy}

More than $90 \%$ of adults with CF have exocrine pancreas insufficiency and require enzyme supplements to aid absorption. ${ }^{1}$ Clinical signs of malabsorption include fatty stools and poor weight gain, but steatorrhoea should be confirmed by direct microscopy for fat globules or by measuring faecal elastase in the stool.

In babies and infants with $\mathrm{CF}$, enteric coated enzyme granules can be administered via a teaspoon at intervals throughout the feed (mixed with a little milk or pureed fruit). Infants and young children require higher doses of pancreatin/kg/body weight than older children and adults. This reflects their higher fat intake $(5 \mathrm{~g}$ fat $/ \mathrm{kg} /$ day compared with the average adult intake of $2 \mathrm{~g}$ fat $/ \mathrm{kg} /$ day). Enzymes have often been prescribed on the basis of one dose for meals and a smaller dose for snacks. However, better control may be achieved by titrating the dose against the fat content of the meal. On this basis most patients require 50-100 units of lipase/g dietary fat/kg/day.

\section{Treatment of gastrointestinal complications in CF}

Mild symptoms of DIOS can be relieved with oral osmotic laxatives, such as lactulose, and adequate hydration. When the abdominal pain is severe and/or accompanied by signs of subacute intestinal obstruction, treatment with gastrografin, $\mathrm{N}$-acetylcysteine, or balanced electrolyte intestinal lavage is necessary.

Drugs to reduce gastric acidity are used frequently in children with CF, usually to help to improve malabsorption, gastro-oesophageal reflux, or epigastric pain. A systematic review has shown limited evidence that these agents provide an improvement in gastrointestinal symptoms and malabsorption. ${ }^{33}$

In the last decade, ursodeoxycholic acid, a naturally occurring hydrophilic bile acid, has been used both for prevention and treatment of liver disease in $\mathrm{CF}^{34}$ There has been evidence from clinical trials that improvement in liver transaminases is seen when ursodeoxycholic acid treatment is used. However, the effectiveness of this treatment in preventing or treating liver disease in CF has not been demonstrated and further clinical trials are needed. Many clinics are now using ursodeoxycholic acid in patients who show elevated liver transaminases or abnormalities on ultrasound suggestive of early liver disease.

\section{REFERENCES}

1 FitzSimmons S. The changing epidemiology of cystic fibrosis. J Pediatr 1993; 122:1-9.

2 Kirk J, Keston M, Mclntosh I, et al. Variation of sweat sodium and chloride with age in cystic fibrosis and normal populations: further investigations in equivocal cases. Ann Clin Biochem 1992;29:145-52.

3 Pollitt R. Screening for cystic fibrosis. Seminars in Neonatology 1998;3:9-15.

4 Wald N, Morris J. Neonatal screening for cystic fibrosis. BMJ 1998;316:404-5

5 Anon. Neonatal screening for cystic fibrosis: position paper. Pediatrics 1983;72:741.

6 Dankert-Roelse J, te Meerman G. Long term prognosis of patients with cystic fibrosis in relation to early detection by neonatal screening and treatment in a cystic fibrosis centre. Thorax 1995;50:712-8.

7 Farrell P, Kosorok M, Laxova A, et al. Nutritional benefits of neonatal screening for cystic fibrosis. N Engl J Med 1997;337:963-9.

8 Merelle ME, Nagelkerke AF, Lees CM, et al. Newborn screening for cystic fibrosis (Cochrane Review). In: The Cochrane Library, Issue 3. Chichester, UK: John Wiley \& Sons, Ltd, 2004.

9 Southern KW. Newborn screening for cystic fibrosis: the practical implications. J R Soc Med 2004;97(suppl 44):57-9.

10 Scott-Jupp R, Lama M, Tanner M. Prevalence of liver disease in cystic fibrosis. Arch Dis Child 1991;66:698-701.

11 Lanng S, Hansen A, Thorsteinsson B, et al. Glucose tolerance in patients with cystic fibrosis: five year prospective study. BMJ 1995;311:655-9.

12 Smyth A, Walters S. Prophylactic antibiotics for cystic fibrosis. In: The Cochrane Library, Issue 3. Chichester, UK: John Wiley \& Sons, Ltd, 2004.

13 McCallum S, Corkhill J, Gallagher M, et al. Superinfection with a transmissible strain of Pseudomonas aeruginosa in adults with cystic fibrosis chronically colonised by $P$ aeruginosa. Lancet 2001;358:558-60.

14 Valerius N, Koch C, Hoiby N. Prevention of chronic Pseudomonas aeruginosa colonisation in cystic fibrosis by early treatment. Lancet 1991;338:725-6.

15 Ryan G, Mukhopadhyay S, Singh M. Nebulised anti-pseudomonal antibiotics for cystic fibrosis (Cochrane Review). In: The Cochrane Library, Issue 3. Chichester, UK: John Wiley \& Sons, Ltd, 2004.

16 Whiteford M, Wilkinson J, McColl J, et al. Outcome of Burkholderia (Pseudomonas) cepacia colonisation in children with cystic fibrosis following a hospital outbreak. Thorax 1995;59:1194-8.

17 Ledson M, Gallagher M, Corkhill J, et al. Cross infection between cystic fibrosis patients colonised with Burkholderia cepacia. Thorax 1998;53:432-6.

18 Cheng K, Smyth RL, Govan JRW, et al. Spread of $\beta$-lactam resistant Pseudomonas aeruginosa in a cystic fibrosis clinic. Lancet 1996;346:639-42.

19 Smyth A, Tan KH-V, Hyman-Taylor P, et al. Once versus three-times daily regimens of tobramycin treatment for pulmonary exacerbations of cystic fibrosis - the TOPIC study: a randomised controlled trial. Lancet 2005;365:573-8.

20 Elphick HE, Southern KW. Antifungal therapies for allergic bronchopulmonary aspergillosis in people with cystic fibrosis (Cochrane Review). In: The Cochrane Library, Issue 3. Chichester, UK: John Wiley \& Sons, Ltd, 2004.

21 van de Schans C, Prasad A, Main E. Chest physiotherapy compared to no chest physiotherapy for cystic fibrosis (Cochrane Review). In: The Cochrane Library, Issue 3. Chichester, UK: John Wiley \& Sons, Ltd, 2004.

22 Thomas J, Cook DJ, Brooks D. Chest physical therapy management of patients with cystic fibrosis. A meta-analysis. Am J Respir Crit Care Med $1995 ; 151: 846-50$. 
23 Cheng K Ashby D Smyth RL Oral steroids for cystic fibrosis (Cochrane Review). In: The Cochrane Library, Issue 3. Chichester, UK: John Wiley \& Sons, Ltd, 2004.

24 Balfour-Lynn I, Walters S, Dezateux C. Inhaled corticoesteroids for cystic fibrosis (Cochrane Review). In: The Cochrane Library, Issue 3. Chichester, UK: John Wiley \& Sons, Ltd, 2004

25 Lands LC, Dezateux C, Crighton A. Oral non-steroidal anti-inflammatroy drug therapy for cystic fibrosis (Cochrane Review). In: The Cochrane Library, Issue 3. Chichester, UK: John Wiley \& Sons, Ltd, 2004.

26 Southern KW, Barker PM, Solis A. Macrolide antibiotics for cystic fibrosis (Cochrane review). In: The Cochrane Library, Issue 3. Chichester, UK: John Wiley \& Sons, Ltd, 2004.

27 Fuchs $\mathrm{H}$, Borowitz D, Christiansen D, et al. Effect of aerosolized recombinant human Dnase on exacerbations of respiratory symptoms and on pulmonary function in patients with cystic fibrosis. N Engl J Med 1994;331:637-42.

28 Jones AP, Wallis CE, Kearney CE. Recombinant human deoxyribonuclease for cystic fibrosis (Cochrane Review). In: The Cochrane Library, Issue 3. Chichester, UK: John Wiley \& Sons, Ltd, 2004.

29 Wark PAB, McDonald V. Nebulised hypertonic saline for cystic fibrosis (Cochrane Review). In: The Cochrane Library, Issue 3. Chichester, UK: John Wiley \& Sons, Ltd, 2004

30 Suri R, Metcalfe C, Lees B, et al. Comparison of hypertonic saline and alternative-day or daily recombinant human deoxyribonuclease in children with cystic fibrosis: a randomised trial. Lancet 2001;358:1316-21.

31 Smyth RL, Walters S. Oral calorie supplements for cystic fibrosis (Cochrane Review). In: The Cochrane Library, Issue 3. Chichester, UK: John Wiley \& Sons, Ltd, 2004

32 Boland M, Stocki D, McDonald N, et al. Chronic jejunostomy feeding with a non-elemental formula in undernourished cystic fibrosis patients. Lancet 1986;ii:232-4

$33 \mathrm{Ng} \mathrm{SM}$, Jones AP. Drug therapies for reducing gastric acidity in people with cystic fibrosis. In: The Cochrane Library, Issue 3. Chichester, UK: John Wiley \& Sons, Ltd, 2004

34 Cheng K, Ashby D, Smyth RL. Ursodeoxycholic acid for cystic fibrosis-related liver disease (Cochrane Review). In: The Cochrane Library, Issue 3. Chichester, UK: John Wiley \& Sons, Ltd, 2004.

\section{ARCHIVIST}

\section{Obesity and the metabolic syndrome}

T he features of the metabolic syndrome include type 2 diabetes, insulin resistance, hypertension, and dyslipidaemia. It is associated with an increase in risk factors for cardiovascular disease. In the US third National Health and Nutrition Examination Survey (NHANES III) of 1988-94, 7\% of overweight, and 29\% of obese, adolescents had the metabolic syndrome. Since that survey the prevalence and degree of obesity have increased in the United States. Now researchers in New Haven and Cincinnati (Ram Weiss and colleagues. New England Journal of Medicine 2004;350:2362-74) have underlined the problem of the metabolic syndrome in obese children and adolescents.

They studied 490 children and adolescents (mean age 12 years), 195 of whom were severely obese (BMI z score > 2.5), 244 moderately obese (z score $2.0-2.5$ ), 31 overweight (BMI 85th to 97th centile), and 20 non-obese (BMI $<85$ th centile). The overweight and non-obese subjects were siblings of obese subjects. Subjects had a standard glucose tolerance test and baseline measurements of blood pressure, plasma lipids, C-reactive protein, interleukin-6, and adiponectin. The metabolic syndrome was defined according to modified national and international criteria, (three or more of: BMI $>97$ th centile, triglyceride $>95$ th centile, HDL cholesterol $<5$ th centile, or impaired glucose tolerance). Insulin resistance was assessed from fasting plasma insulin and glucose using a homeostatic model.

The metabolic syndrome was diagnosed in 39\% of moderately obese and $50 \%$ of severely obese subjects but in none of the overweight or non-obese siblings. Among obese subjects the risk of the metabolic syndrome increase by $55 \%$ for each increase of 0.5 in BMI z score and by $12 \%$ for each unit increase in insulin resistance. Increasing obesity was associated with increasing prevalence of impaired glucose tolerance, increasing insulin resistance and systolic blood pressure, and increasing blood concentrations of glucose, insulin, triglycerides, C-reactive protein, and interleukin-6. Concentrations of HDL cholesterol and adiponectin decreased with increasing obesity. All of these changes are associated with increased cardiovascular risk in adult life.

Seventy-seven obese subjects, 34 of whom had the metabolic syndrome at the initial assessment, were followed up after an average of 22 months. Eight had developed type 2 diabetes. Ten of the 34 no longer satisfied criteria for the metabolic syndrome but 16 of 43 subjects who did not have the syndrome initially now satisfied the criteria.

The metabolic syndrome is common among obese children and adolescents and increases in prevalence with increasing obesity. Risk factors for adult cardiovascular disease are already present with obesity at this age. 